\title{
From profile to sawtooth control: developing feedback control using ECRH/ECCD systems on the TCV tokamak
}

\author{
J I Paley, F Felici, S Coda, T P Goodman and the TCV Team \\ Ecole Polytechnique Fédérale de Lausanne (EPFL), Centre de Recherches en Physique des Plasmas, Association \\ Euratom-Confédération Suisse, CH-1015 Lausanne, Switzerland \\ Email: james.paley@epfl.ch
}

\begin{abstract}
Real time control of heating systems is essential to maximise plasma performance and avoid or neutralise instabilities under changing plasma conditions. Several feedback control algorithms have been developed on the TCV tokamak that use the electron cyclotron (ECRH/ECCD) system to control a wide range of plasma properties, including the plasma current, shape, profiles as well as the sawtooth instability. Controllers have been developed to obtain sawteeth of a pre-determined period, to maximise the sawtooth period using an extremum seeking control algorithm and finally to provide simultaneous control of the plasma emission profile peak and width using multiple independent EC actuators.
\end{abstract}

Pacs numbers. 52.50.Sw 52.55.-s 52.55.Fa 52.55.Tn 52.55.Wq 07.05.Dz

Submitted to. Plasma physics and controlled fusion

\section{Introduction}

Electron cyclotron (EC) resonance heating (ECRH) \& current drive (ECCD) systems are used for plasma heating, controlling instabilities such as sawteeth and neoclassical tearing modes (NTM), and tailoring profiles in plasmas. They provide a heat and current deposition profile in the plasma that is highly localised in radial position, with the ability to shift the deposition on demand by adjusting the EC launcher injection angle. There are also no constraints on coupling the EC wave from the launcher to the plasma, as it propagates in vacuum. There may however be unexpected variations in plasma conditions. For this reason, especially in a future fusion reactor, real time feedback controllers are required which react to the changing plasma conditions, changing the launcher angles and injected power levels as required. This has motivated the development of feedback control systems, algorithms and experiments to demonstrate control of instabilities and profiles using the ECRH \& ECCD systems.

Several tokamaks have implemented feedback controllers using their EC systems. DIII-D has demonstrated using ECCD at constant injection angle using the toroidal magnetic field and rigid shifts of the plasma position to change the deposition location to "search and suppress" NTMs [1]. JT-60U and ASDEX-U have also carried out real time control of NTMs, by modulating the EC beam power in an attempt to drive current in the O-point (and only the O-point) of the island [2,3], as the island spins toroidally past the deposition location. JT-60U has demonstrated control of the NTM by actuating the 
EC launchers [4]. Finally, Tore Supra used EC launchers in a feedback control loop to shorten the sawtooth period of fast ion stabilised sawteeth [5].

The sawtooth instability is a magnetohydrodynamic instability that limits the core plasma pressure, and may also induce an NTM which in turn degrades the plasma confinement, possibly even resulting in a rapid, total loss of the plasma discharge in a disruption [6,7]. The sawtooth crash is largely determined by the magnetic shear at the $\mathrm{q}=1$ surface [8] and the EC systems may be used to tailor the magnetic shear, in order to delay or expedite the onset of the crash, hence increasing or decreasing, respectively and as required, the sawtooth period. TCV has recently demonstrated feedback control of the sawtooth period by actuating the EC launcher injection angle [9], providing the ability to set the sawtooth period to a pre-determined value which must be less than the maximum sawtooth period possible. This work has now been extended by the development and successful application of an alternative algorithm, which seeks to maximise, rather than set, the sawtooth period.

The ability to control plasma profiles, eg pressure, density, current, temperature etc is important in obtaining high performance plasmas and managing the bootstrap current while avoiding MHD activity, generating and maintaining internal transport barriers and impurity accumulation [10]. Recent TCV experiments have started to address the issues of using the ECRH/ECCD systems for profile control, where multiple EC beams, deposited in different locations in the plasma, are used to control several parameters of the profile simultaneously. This is a multi-input-multi-output (MIMO) control problem and lends itself naturally to the use of state-space model based controller design techniques as previously explored in [10].

This paper is organised as follows: The experimental setup will first be described with a review of the TCV EC systems and real time capabilities. We shall then provide a concise review of the recent TCV real time feedback control experiments. This will be followed by a detailed report on the latest experiments with feedback control loops using one and more EC beams to control parameters of the plasma emission profile (soft x-ray) using MIMO control methodology and experiments to maximise the sawtooth period.

\section{Experimental setup}

The Tokamak a Configuration Variable (TCV) [11] (major radius $0.88 \mathrm{~m}$, minor radius $0.255 \mathrm{~m}$, height $1.5 \mathrm{~m}$, elongation 0.9 to 2.8 , maximum plasma current $1 \mathrm{MA}$, maximum toroidal magnetic field $1.54 \mathrm{~T}$, Ohmic heating power $\sim 1 \mathrm{MW}$ ) has a $4.5 \mathrm{MW}$ real time steerable multiple launcher ECRH/ECCD system as the dominant auxiliary heating system. Through the wide range of achievable poloidal and toroidal angles, it is possible to heat and/or drive current in a wide range of plasma configurations. There are two ECRH/ECCD subsystems on TCV operating at the $2^{\text {nd }}$ and $3^{\text {rd }}$ harmonic $(82.7 \mathrm{GHz}-\mathrm{X} 2$ and $118 \mathrm{GHz}-\mathrm{X} 3)$. 


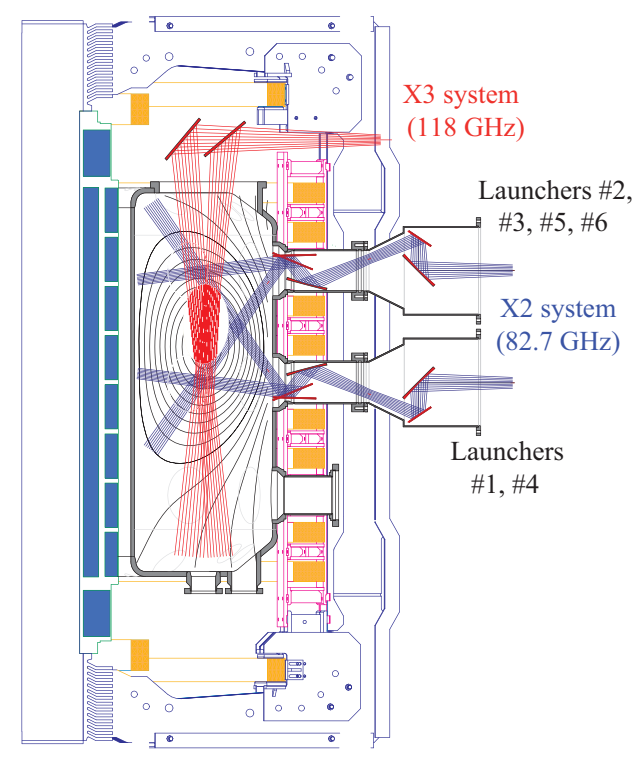

Figure 1. Diagram of the X2 and X3 EC launcher injection showing the upper and equatorial X2 EC injection ports, the top launched X3 beam and a range of injection trajectories (excluding plasma refraction). A typical plasma equilibrium is also shown.

\subsection{The X2 EC subsystem}

The main X2 EC subsystem [12] consists of 6 gyrotrons, waveguides, matching optical units and independent launchers, in addition to a power supply for each cluster of 3 gyrotrons. The X2 system has a cut-off in plasma density at $\mathrm{n}_{\mathrm{e}}=4.2 \times 10^{19} \mathrm{~m}^{-3}$. The launchers inject EC into 4 upper and 2 midplane ports of the TCV vacuum vessel (Figure 1), distributed over 4 toroidal locations. Each launcher can be rotated on its longitudinal axis between each plasma shot and the final mirror in the launcher is controllable in real time throughout the shot. At the nominal longitudinal angle ( 0 or 180 degrees) the EC is injected along the major radial-vertical plane into the plasma providing almost entirely current-drive free ECRH. At 90deg longitudinal angle, the EC wave is injected into the major radial-toroidal plane generating ECCD. The full range of intermediate longitudinal angles are also available providing varying parallel wave numbers at different radial locations as required. The final mirror provides real time control over the deposition location.

The 2 Regulated High Voltage Power Supplies (RHVPS) provide voltage for each gyrotron cathode. The voltage amplitude ( 0 to $69 \mathrm{kV}$ ) on the cathode determines the power of the EC output beam ( 0 to $500 \mathrm{~kW}$ ), according to a calibration curve. Each RHVPS is supplied with a voltage reference signal, either by pre-programmed waveform generators or by the real time control system. There are some limitations in the range of accessible cathode voltages. Below an output power of $190 \mathrm{~kW}(59 \mathrm{kV})$, the gyrotron does not efficiently generate the EC wave and the beam dump will heat excessively. Therefore below this limit, the cathode voltage must go to zero or hold at $59 \mathrm{kV}$. Also the ramp must not be faster than 0 to $69 \mathrm{kV}$ in $700 \mu \mathrm{s}$. These limitations are built into the controller logic (as a Simulink block containing the power-voltage calibration) to prevent these limits being exceeded.

\subsection{Real time control hardware, diagnostics and algorithm development}

The real time controller is based on multi-channel analogue acquisition modules linked to high performance PCs as described in [13]. Programming for the real time control system is done entirely in Simulink ${ }^{\circledR}$ and the real time workshop for embedded targets, providing the ability to quickly generate, simulate and test algorithms, as well as generate real time $\mathrm{C}$ code and compile for the 
hardware. This controller is able to run on a $100 \mathrm{kHz}$ control cycle, typically operating at $10 \mathrm{kHz}$ for the sawtooth and profile control experiments described in this paper. The main diagnostic used to construct observers for the algorithms described in this paper is a soft X-ray diagnostic, the Duplex Multiwire Proportional X-ray system (DMPX) [14]. This detector is made up of two superimposed wire-chambers sensitive to soft X-ray emission with energy between 3 and $30 \mathrm{keV}$ and measures the line integrated emission from the plasma across 64 vertically orientated lines of sight (with the camera observing from the bottom of the vacuum vessel). The mean distance separating each line of sight is $7.9 \mathrm{~mm}$ at the equatorial plane of the vacuum vessel. The data from each line of sight is amplified and acquired by the real time control system. The actuator commands are provided by an analogue output module which generates the reference signals for the EC launchers and power supplies.

\section{Overview of previous TCV EC control experiments}

Several real time control experiments have previously been carried out at TCV using the EC actuators to control the plasma current, shape, emission profile peak and the sawtooth period. A short overview of these experiments is provided in this section.

\subsection{ECCD plasma current control}

The plasma current on TCV may be driven entirely non-inductively, using ECCD, with the Ohmic coils set to provide zero loop voltage during the ECCD phase. Typically these experiments provide no control over the magnitude of the plasma current and therefore a real time feedback controller was implemented to control the amplitude of the plasma current by actuating the ECCD power [15] (ie gyrotron cathode voltage provided by the RHVPS). A simple analogue proportional-integral (PI) controller was used and was successfully able to track the target reference current.

\subsection{ECRH plasma shape control}

TCV can produce highly elongated plasmas $(\kappa>2)$. At low current the vertical instability limits the elongation, but by broadening the plasma current profile eg using off-axis ECRH, the stability is improved and highly elongated $(\kappa \sim 2.4)$, low current $\left(\mathrm{I}_{\mathrm{N}} \sim 1\right)$ plasmas can be obtained. [16]. In order to avoid the stability limit, the plasma magnetic shaping field is held constant and the plasma current profile is broadened using off-axis ECRH, leading directly to an elongation. Using a real time estimation of the plasma elongation from flux measurements at fixed points outside the nominal plasma edge as described in [15] and a PI controller, the EC power was used to control the magnitude of the elongation and the EC launchers were moved in feedback to maintain the absorption as far as possible at constant normalized radius.

\subsection{Peak-in-profile control}

The signals from the high resolution 64 channel soft x-ray diagnostic (DMPX) were used to generate a real time emission profile, using a cubic spline fit on the calibrated data. Initially the profile peak was used in a feedback control loop, actuating an EC launcher to control the peak amplitude [13], eg moving the launcher for more centralized EC deposition to increase the profile peak. This algorithm has now been further developed to simultaneously control the width and the amplitude of the peak using multiple actuators and a state-space controller and is discussed in section 4.

\subsection{Maximising X3 EC absorption}

To heat higher density plasmas, TCV has an X3 EC subsystem which can heat plasmas with electron densities up to $n_{e}<11.5 \times 10^{19} \mathrm{~m}^{-3}$. The X3 EC wave is only weakly absorbed in the plasma and is therefore injected from the top of the vacuum vessel into the plasma (see Figure 1), with the aim to maximise the path length along the resonance layer. An extremum seeking controller was implemented for the X3 launcher injection angle in order to maximise the first-pass absorption in the 
plasma [17]. As the effect of dynamically varying density and temperature profiles is to unpredictably shift the deposition relative to this layer, a real time controller was implemented, adjusting the angle of EC injection to maximise the temperature of the plasma. This is a similar approach to the sawtooth period maximisation algorithm discussed later in this paper (section 5) and consists of applying a small oscillation to the injection angle, observing the resulting plasma temperature oscillation (at the same frequency as that of the applied oscillation) and shifting the average position of the launcher in the direction corresponding to increasing temperature.

\subsection{Sawtooth control}

The sawtooth period was successfully controlled to match a pre-determined reference period using a real time feedback control loop [9], acting on the X2 EC launcher injection angle to tailor the plasma current profile in the vicinity of the $\mathrm{q}=1$ surface. This controller used a sawtooth crash detection algorithm for the sawtooth period observer and compared with a target reference period to generate the error signal. A simple PI controller was used, together with a gain switch to adapt the controller gain to the deposition location based on predetermined conditions. This was required due to the very small angular range under which the sawtooth period responds to changes in the injection angle. In particular, when depositing EC power near $\mathrm{q}=1$, the sawtooth period response becomes very strong, i.e., even small adjustments to the launcher can substantially change the sawtooth period. The controller was successfully able to generate sawteeth with a range of periods. If the target period is too near the maxima in the sawtooth period, the controller will become unstable. In order to obtain the maximum, an alternative controller is required. This has been developed and is discussed in section 5 .

\section{Profile control}

As direct measurements of the plasma temperature and current profiles are not yet available in realtime on TCV, experiments were carried out to control the line-integrated soft X-ray emission profile from the DMPX diagnostic (see section 2.2). The signal given by this diagnostic, when the plasma density and effective charge are constant, provides an indication of the plasma temperature profile.

Feedforward experiments were carried out to characterize the response of the soft X-ray profile to changes in deposited power at different locations. Two ECRH beams were directed into the plasma at constant injection angle, corresponding to $\rho=0.2$ and $\rho=0.5$. The power of each of these two beams was modulated by $100 \mathrm{~kW}$ using two independent pseudo-random binary noise sequences. A spline fit was used to fit the data with the peak defined as the maximum in the fitted profile and the width defined as the sum of the fitted profile divided by the peak. Although other choices for parameterization of the profile are possible, these were chosen in initial trials for simplicity.

A system identification algorithm was used to determine a second-order state space model approximately describing the plasma response to the ECRH power modulations. The identified model was then used as basis for the design of an LQG (linear-quadratic-gaussian) controller, consisting of a Kalman state observer and feedback of the estimated states. The Kalman filter provides an estimate of the state using knowledge of the model and statistical properties of the noise. The state feedback matrix is designed using an LQR (linear-quadratic regulator) approach, minimizing a cost function which penalizes state errors and controller effort. This is a well-known technique in the control engineering field described in textbooks such as K Åström [18].

The resulting controller was implemented in Simulink and an initial test carried out on TCV plasmas as shown in Figure 2. During these tests limitations were placed on the controller in order to prevent requests for very large, fast swings in the gyrotron power. The controller has feedforward, reference and feedback terms, with the feedforward term in proportion to the reference. A limit was placed on 
the feedback term, allowing a maximum of $+/-100 \mathrm{~kW}$ deviation from the feedforward and therefore the power quickly saturates at the beginning and end of the control phase of Figure 2, limiting the ability to fully obtain the provided references.

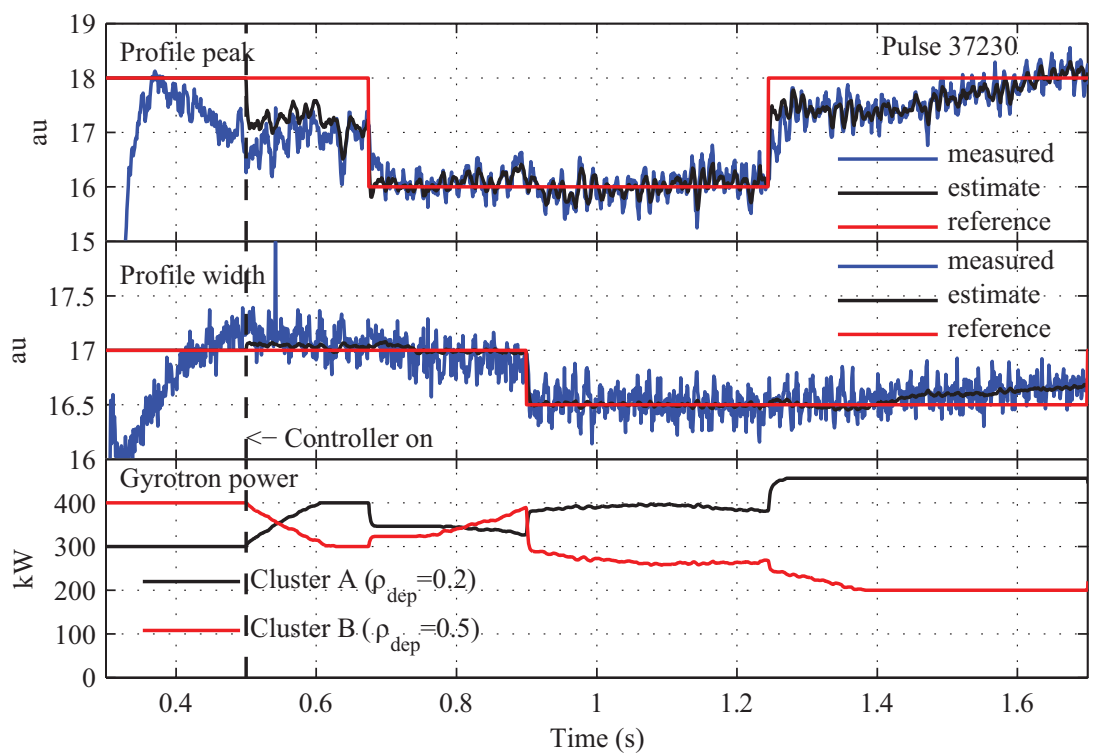

Figure 2. Feedback control of the plasma emission profile demonstrating simultaneous control of the profile peak and width using two independent gyrotrons. As a preliminary result, this experiment demonstrates control of the profile peak and width, despite the limits enforced on the power of the feedback term (causing the saturation in power of both gyrotrons at $t=0.6-0.7$ and $t \sim 1.3$ to the end of the shot). The profile width and peak are successfully controlled between 0.7 and $1.25 \mathrm{~s}$. After this time, the prescribed values are not obtained due to saturation of the actuators. The peak cannot be obtained as the central gyrotron is already at maximum power and the width cannot be reduced as the off-axis gyrotron is at minimum power. A small, steady increase in the plasma current from $1.2 \mathrm{~s}$ causing the peak and width to increase, is balanced by the reduction in power of the off-axis gyrotron until it saturates at $1.4 \mathrm{~s}$.

Further work is planned on this topic and will address a more appropriate choice of basis functions, as well as techniques to control both the deposition power and deposition location simultaneously and the controller limitations discussed above will be relaxed to allow control of the full range of gyrotron power.

\section{Maximising the sawtooth period using a extremum seeking controller}

In order to maximise the sawtooth period, the current profile must be tailored such that during the sawtooth ramp phase, the critical current gradient at the $\mathrm{q}=1$ surface is achieved as late as possible. This is done by injecting current (directly through co-current EC injection and/or indirectly through EC heating and thus conductivity increase) immediately outside the $\mathrm{q}=1$ surface, or counter-current immediately inside the $\mathrm{q}=1$ surface in order to flatten the current profile. As neither the position of the $\mathrm{q}=1$ surface nor the deposition location is known in real time on TCV, it is necessary to build a controller that relies only upon the observed sawtooth period and the actuator position. The scheme used here was to oscillate the launcher such that the EC deposition moves in the region of the $\mathrm{q}=1$ surface, observe the response of the sawtooth period and move the mean position of the launcher in the direction of increasing sawtooth period. This is an extremum control, or self-optimization control 
algorithm [19] and is similar to the X3 absorption maximisation discussed in section 3.4 [17]. Extremum control is also proposed to optimise the coupling of the lower hybrid radiofrequency antenna and plasma on the Frascati Tokamak Upgrade [20] and similar techniques could be used to minimise the period of fast ion stabilized sawteeth in future devices.

Figure 3 shows a block diagram of the algorithm.

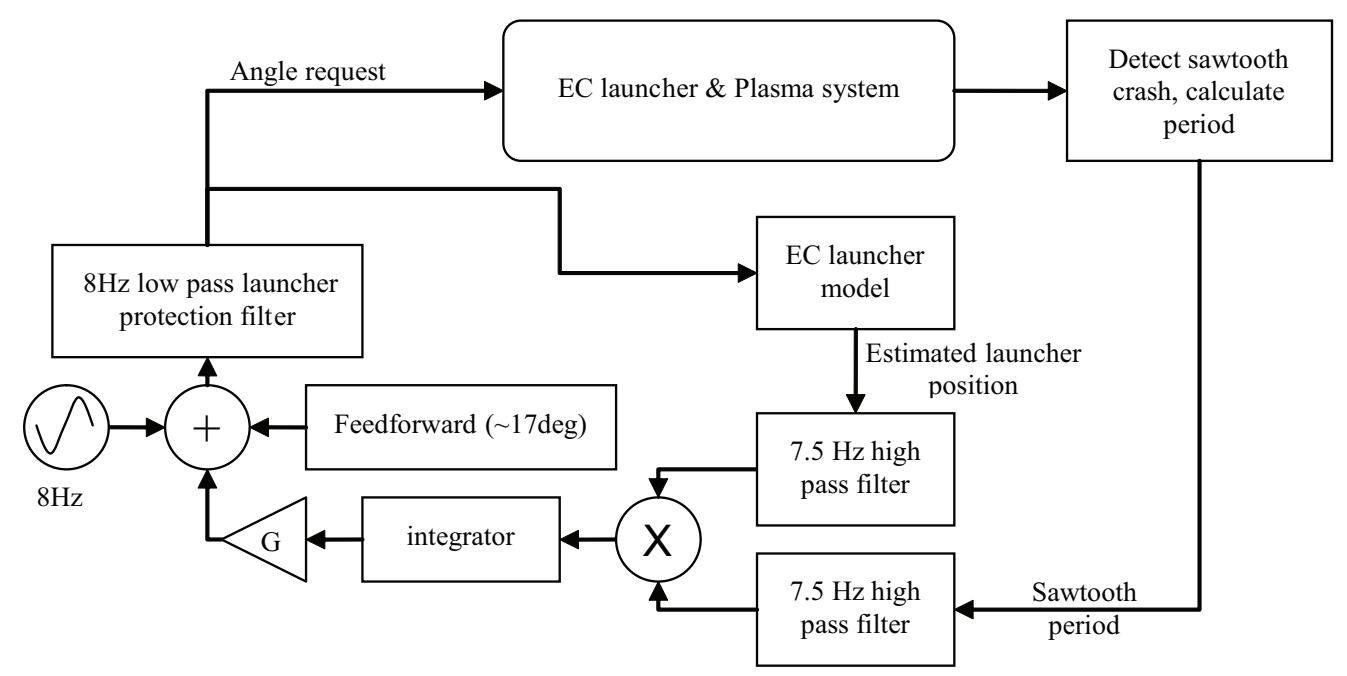

Figure 3. Block diagram of the sawtooth period maximum seeking algorithm.

The launcher is oscillated at $8 \mathrm{~Hz}$ with amplitude of 1 degree. The sawtooth crash is first detected using the method described in [9], by looking for a large negative derivative in the core plasma soft xray emission which correspond to a sawtooth crash and is reliable over a wide range of sawteeth periods, crash sizes and sawtooth shapes, with only occasional errors. A high pass filter operates on the sawtooth period observer to obtain the response at this frequency. The signal is then multiplied by the estimated launcher position, calculated using the EC launcher model using the requested angle (after passing through an identical high pass filter to match the phase as closely as possible). This gives a signal which is proportional to the rate of change of the sawtooth period with the launcher angle. Typically the signal would now be passed through a low pass filter, with cut-off $<<8 \mathrm{~Hz}$ to obtain only the DC component, however this was found not to be essential as the launcher protection filter of $8 \mathrm{~Hz}$ cut-off would in any case remove the highest frequency components and an additional filter would impact on the phase of the system. The integrator and gain $(G)$ are then applied before addition of a feedforward angle and the sinusoid. An obligatory $8 \mathrm{~Hz}$ low pass launcher protection filter is added before the analogue output signal is generated.

The algorithm was first tested on simple simulations of the plasma sawtooth response, built using previous feedforward sweeps of the EC deposition across the $\mathrm{q}=1$ surface as described in [9]. Initial simulations showed the controller gains were difficult to select for the cases when the deposition is far from q=1 as well as very close, and a gain switch was added. In this way, when the sawtooth period increases above a pre-determined threshold (typically $5-8 \mathrm{~ms}$ in these experiments), the controller gain $\mathrm{G}$ is reduced (by between $5-10$ ), allowing for the system to quickly move the deposition to near the $\mathrm{q}=1$ surface and then take minor corrections to maintain the deposition in the optimum location.

As the controller gains are deduced from feedforward sweeps of EC deposition across the $q=1$ surface, they are specific to the chosen plasma configuration. For different plasma shapes, current etc, 
the model of the plasma sawtooth response should be first updated using a feedforward sweep on the new configuration and then simulations of the control loop can be used to optimise the controller gains.

Figure 4 shows the maximisation algorithm applied to a sawtoothing plasma shot. EC is switched on with dominant ECRH at time $0.3 \mathrm{~s}$ and the plasma heats. The controller is activated at $0.4 \mathrm{~s}$ with the initial launcher position at an angle of $17 \mathrm{deg}$, corresponding to depositing power outside the $\mathrm{q}=1$ surface at normalized radius $\sim 0.52$. The controller almost immediately requests the launcher to move to larger angle until the maximum of $12 \mathrm{~ms}$ sawteeth is obtained at $\sim 0.6 \mathrm{~s}$. The launcher remains approximately in the same location until a disturbance is added at $0.9 \mathrm{~s}$ : the plasma vertical position is moved $2 \mathrm{~cm}$ down in $80 \mathrm{~ms}$ and almost immediately small, irregular sawteeth are obtained, corresponding to the deposition moving inside the $\mathrm{q}=1$ surface. The controller quickly responds by moving the launcher to smaller angle until the large sawteeth are again obtained. There is a reduction in the maximum sawtooth period obtained over the flattop phase (12ms to $9 \mathrm{~ms})$. This is likely to be due to changing plasma conditions throughout the shot and is typical of these plasmas.

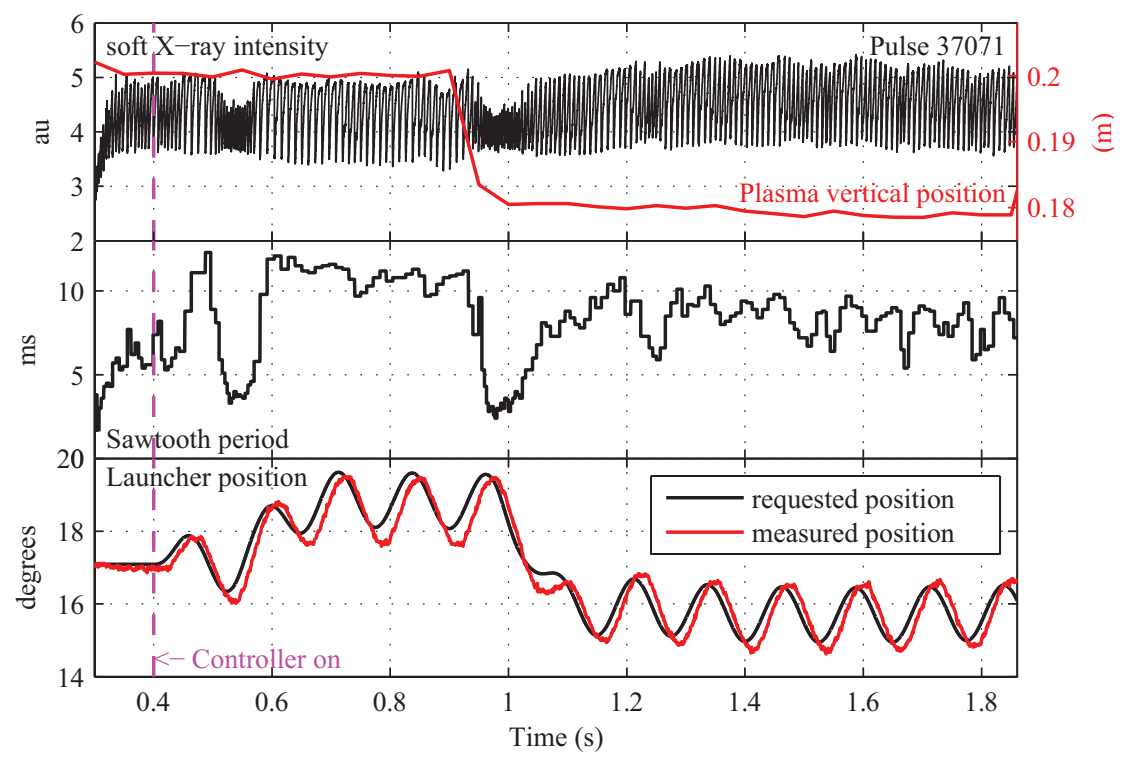

Figure 4. Pulse 37071. Maximising the sawtooth period. The controller is able to obtain and track the maximum sawtooth period, despite the shift in the plasma vertical position at $0.9 \mathrm{~s}$.

The difficulties in this approach lie in the initial position of the launcher - too far away from the $q=1$ surface and the sawteeth may not respond at all to the oscillation and therefore the controller would not know in which direction to move the launcher. However, as the technique simply relies on the plasma response, once the sawtooth response as a function of launcher angle has been determined in a feedforward scan, the controller gains and feedforward launcher position can be determined and subsequently tested in a simulation of the control loop before final implementation. The effect of the oscillation itself acts to both move the deposition away from the optimal location and perhaps also to broaden the deposition profile, which would inhibit the ability to obtain the longest sawtooth period. To mitigate this effect one possibility to be explored would be for the controller to reduce the amplitude of the oscillation instead of the gain $G$ when larger sawteeth are obtained. Further development of this algorithm would involve optimising the gains, oscillation frequency, and filters. 


\subsection{Using 2 EC beams to maximise the sawtooth period}

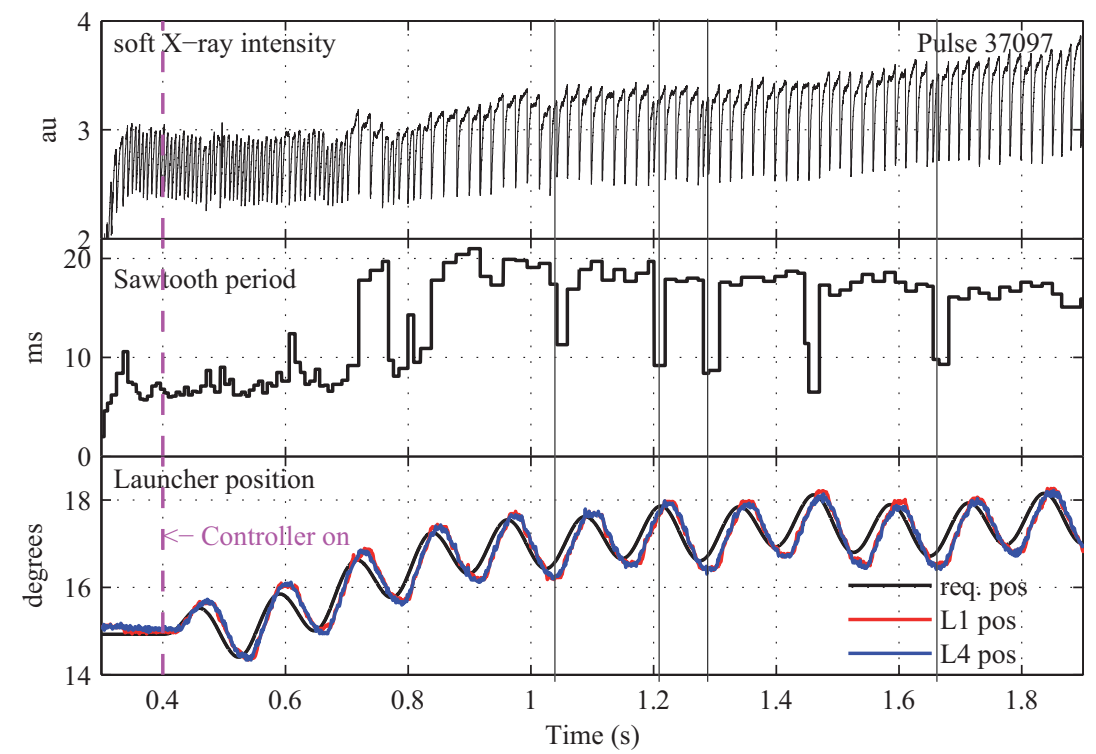

Figure 5. Pulse 37097. Using two launchers (labelled L1 and L4) to maximise the sawtooth period. The controller finds and maintains the peak in the sawtooth period. There are occasional bursts of sawteeth with a shorter period. These seem to correspond with both extrema in the launcher oscillation, indicated by the vertical lines, demonstrating that the EC deposition is optimised on the peak in the sawtooth period. Note there is a false detection of a sawtooth crash at $\mathrm{t}=1.44 \mathrm{~s}$ leading to the sudden decrease in the real time calculation of the sawtooth period at this time.

The maximum effect on the local current profile arises when multiple beams are precisely aligned to deposit power/co-current at the same location. In TCV the launchers are aligned by mechanical measurements and by sweeping the EC deposition from each launcher across the $q=1$ surface to compare the angle versus sawtooth period curve for each launcher. These techniques provide a quoted alignment accuracy of 0.2 degrees [21]. The ability to simultaneously control multiple launchers is important; however it was not clear that whilst under feedback control, the launchers could be controlled with sufficient accuracy. The dynamics of the mechanical launchers may lead to an inability to align the beams. The maximisation algorithm from the previous section was applied to actuate 2 equatorial X2 launchers simultaneously with the aim to generate the longest possible sawteeth throughout the plasma flattop phase, relying upon the alignment above to ensure both launchers are depositing at the same location as far as possible. The controller was able to find and track the longest sawteeth, having a period $>20 \mathrm{~ms}$ at the beginning of the plasma discharge, reducing to $18 \mathrm{~ms}$ towards the end of the shot as shown in Figure 5. There are occasional shorter sawteeth, which seem to correlate with both positive and negative extrema in the sinusoidal oscillation; as is expected when the deposition moves too far from the optimal location, demonstrating the oscillation is taking place at/near the peak in the sawtooth period. In this experiment, there are almost no sawteeth with periods within the range of 12 to $18 \mathrm{~ms}$, leading to a discontinuity in the launcher angle versus sawtooth period curve, which is not seen in the experiment with 1 EC beam (Figure 4). The sawteeth also have a different shape [22], with a triangular shape seen in the sawteeth of Figure 4 and a saturated shape in Figure 5. The crash phase of the occasional short sawteeth of Figure 5 occurs just after the saturation in the ramp is obtained, at approximately $50 \%$ of the period of the longer sawteeth. When the EC is deposited away from the optimal location, the crash is triggered at the start of the saturation phase, leading to the $50 \%$ drop in the sawtooth period with no intermediate values. 


\section{Summary}

A wide range of techniques and algorithms have been applied for the feedback control of the plasma current, shape, profile and sawtooth period by controlling, in real time, the ECRH/ECCD power and launcher angles. A feedback controller that maximises the sawtooth period has been implemented and is able to obtain and track the maximum in the period by adjusting the EC launcher angle, even when a disturbance such as a shift in the vertical position of the plasma is applied. A demonstration of simultaneous control of $2 \mathrm{EC}$ launchers to maximise the sawtooth period was also successful. Further developments using MIMO linear control methodology for simultaneous control of the plasma emission profile peak and width using multiple independent EC actuators have also been presented.

\section{Acknowledgments}

This work was funded in part by the Swiss National Science Foundation.

\section{References}

[1] La Haye RJ et al 2002 Physics of Plasmas 92051

[2] Isayama A et al 2009 Nucl. Fusion 49055006

[3] Maraschek M et al 2007 Phys. Rev. Lett. 98025005

[4] Isayama A et al 2003 Nucl. Fusion 431272

[5] Lennholm M et al 2009 Phys. Rev. Lett. 102115004

[6] Sauter O et al 2002 Phys. Rev. Lett. 88105001

[7] Gude A et al 2002 Nucl. Fusion 42833

[8] Porcelli F et al 1996 Plasma Phys. Control. Fusion 382163

[9] Paley JI et al 2009 Plasma Phys. Control. Fusion 51055010

[10] Moreau D et al 2008 Nucl. Fusion 48106001

[11] Hofmann F et al 1994 Plasma Phys. Control. Fusion 36 B277

[12] Goodman TP and the TCV team 2008 Nucl. Fusion 48054011

[13] Paley JI et al 2009 Nucl. Fusion 49085017

[14] Sushkov A et al 2008 Rev. Sci. Instrum. 79023506

[15] Paley JI et al 2007 Plasma Phys. Control. Fusion 49 1735-1746

[16] Pochelon A et al 2001 Nucl. Fusion 45 1224-31

[17] Alberti S et al 2005 Nucl. Fusion 45 1224-1231

[18] Åström K \& Wittemark B (1997) Computer Controlled Systems, 3rd ed, Prentice Hall

[19] Krstic M \& Wang H 2000 Automatica 36 596-601

[20] Carnevale D et al 2009 Fusion Engineering and Design 84 554-558

[21] Goodman TP et al 1999 Proceedings $26^{\text {th }}$ EPS CCFPP 1999 ECA Vol 23J $1101-1104$

[22] Furno I et al 2001 Nucl. Fusion 41 403-420 